\title{
имитационное моделирование многофазных многокомпонентных систем в потоке газа
}

П.А. Сеченов ${ }^{1}$, к.m.н., pavesa89@mail.ru

И.А. Рыбенко 1, д.m.н., доиент, rybenkoi@mail.ru

В.П. Цьилбал 1, д.m.н., npoфpeccop, tsymbal33@mail.ru

1 Сибирский государственный индустриальный университет, 2. Новокузнеик, 654007, Россия

В статье рассмотрена имитационная модель физико-химических процессов в колонном струйноэмульсионном реакторе. В таблицах представлены исходные составы входных продуктов: железной руды, извести, угольного топлива.

Вычислительный эксперимент проводился с помощью имитационной модели методом частиц. Предложена методика взаимодействия частиц в колонном реакторе, подробно рассмотрены механизмы взаимодействия и изменения состава газа по высоте реактора. В качестве языка программирования выбран Action Script 3.0, позволяющий анимировать движение частиц и наглядно изучать происходящие процессы. Он компилировался в среде программирования Adobe Animate. Реализован механизм горения угольного топлива, учтены варианты выгорания летучих в угольном топливе в зависимости от наличия кислорода в слое газа. В частицах угольного топлива по мере выгорания динамически изменяются свойства: уменьшается размер, изменяется состав и увеличивается плотность. Для учета изменения состава газа по высоте колонного реактора реализован соответствующий класс, предложен механизм подъема газа и изменения состава газа в виде слоев по высоте реактора.

В результате моделирования было выявлено, что при выгорании угольного топлива и изменении свойств частицы начинают подниматься вверх по колонному реактору. Наибольшая концентрация мелких частиц угольного топлива наблюдается чуть ниже уровня шлаковой летки, куда вылетают частицы шлака и угольного топлива.

Моделирование показало, что концентрация кислорода резко падает уже на первом метре по высоте реактора, концентрация монооксида углерода незначительно снижается, а диоксида углерода повышается.

Разработанная и реализованная модель позволит оптимально управлять сырьевыми и топливноэнергетическими ресурсами в металлургическом производстве, для чего в дальнейшем потребуется учитывать процессы нагрева, плавления веществ в колонном реакторе, а также эндотермические и экзотермические реакции на уровне частиц.

Ключевые слова: колонный реактор, термодинамика, имитационная модель, взаимодействие частии, алгоритм, программная реализаиия.

В статье показан пример создания программного комплекса для решения задач моделирования физико-химических процессов в колонном струйно-эмульсионном реакторе. Колонный реактор, схема которого подробно рассмотрена в [1], представляет собой технологический агрегат для реализации процессов восстановления железа в агрегате струйноэмульсионного типа. Исходными материалами при организации восстановительного процесса являются железная руда, известь и угольное топливо, конечными продуктами - металл, шлак и отходящий газ. Состав исходных шихтовых материалов приведен в таблицах 1-3.

\section{Механизмы взаимодействия частиц}

Представим принципы взаимодействия между частицами, реализованные в имитаци- онной модели колонного струйно-эмульсионного реактора. Каждые две секунды в колонный реактор попадают частицы железной руды с радиусом от 0,1 до 2 мм, распределенные по нормальному закону. Алгоритм генерации нормального закона распределения частиц рассмотрен в статье [2]. Зависимость скорости расплавления частицы железной руды от радиуса соответствует принятой в работах $[3,4]$. Скорость горения угольного топлива, от которой зависят состав газа и распределение теплоты по высоте колонного реактора, взяты из работ [5-8].

В имитационной модели также реализованы механизмы:

- упругих и неупругих столкновений частиц с расчетом скоростей после столкновения;

- объединения частиц, в результате чего образуется новая частица, средний состав и 
Состав железной руды

Table 1

Iron ore composition

\begin{tabular}{|l|c|c|c|c|c|c|c|c|c|c|c|}
\hline Компонент & $\mathrm{FeO}$ & $\mathrm{MnO}$ & $\mathrm{SiO}_{2}$ & $\mathrm{CaO}$ & $\mathrm{MgO}$ & $\mathrm{Al}_{2} \mathrm{O}_{3}$ & $\mathrm{P}_{2} \mathrm{O}_{5}$ & $\mathrm{TiO}_{2}$ & $\mathrm{~V}_{2} \mathrm{O}_{5}$ & $\mathrm{Cr}_{2} \mathrm{O}_{3}$ & $\mathrm{~S}$ \\
\hline Содержание, \% & 62,50 & 0,20 & 24,00 & 0,35 & 0,08 & 11,40 & 0,28 & 0,55 & 0,36 & 0,10 & 0,14 \\
\hline
\end{tabular}

Таблица 2

Состав извести

Table 2

Lime composition

\begin{tabular}{|l|c|c|c|c|c|c|}
\hline Компонент & $\mathrm{SiO}_{2}$ & $\mathrm{CaO}$ & $\mathrm{MgO}$ & $\mathrm{Al}_{2} \mathrm{O}_{3}$ & $\mathrm{H}_{2} \mathrm{O}$ & $\mathrm{CO}_{2}$ \\
\hline Содержание, \% & 2,00 & 90,00 & 1,80 & 1,00 & 1,00 & 4,00 \\
\hline
\end{tabular}

Состав угольного топлива

Таблиия 3

Coal fuel composition

Table 3

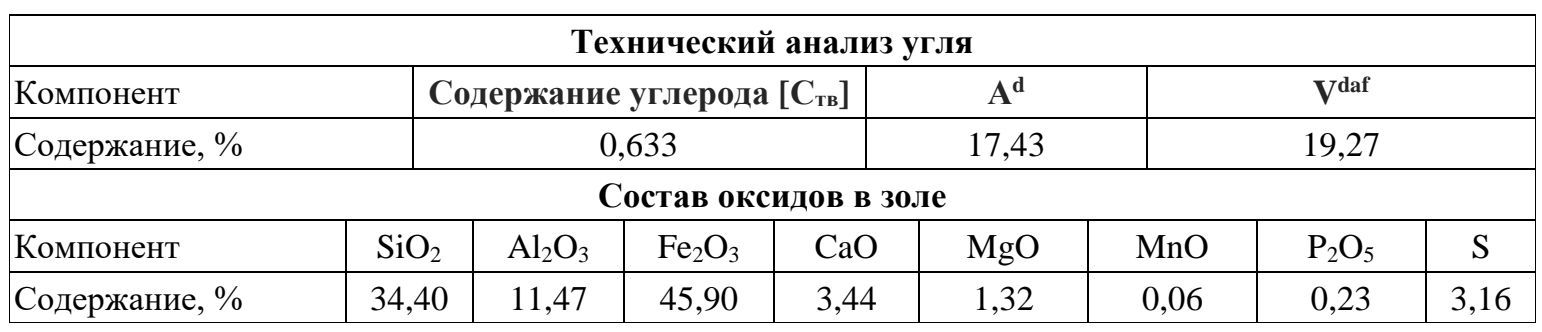

Примечание: $A^{d}$ - зольность (ГОСТ 11022-95); V daf - выход летучих вещчеств (ГОСТ 6382-92).

плотность которой принимаются в соответствии с балансовыми расчетами;

- разделения частицы на составляющие после полного расплавления;

- изменения плотности частицы в результате встречи с газом;

- выгорания частицы с постепенным уменышением ее радиуса и выделением газообразных продуктов.

Состав многофазной многокомпонентной системы неоднородный, поэтому для описания физико-химических механизмов в разработанную имитационную модель добавлены классы, позволяющие учитывать концентрации конденсированных веществ и состава газов по высоте колонного реактора.

\section{Выбор языка программирования}

Язык программирования для реализации модели - Action Script 3.0, среда программирования - Adobe Animate 2021. Программы, написанные на языке Action Script, дают возможность лучше понять процесс за счет анимации моделируемых явлений [9]. Также этот язык является кроссплатформенным, что позволяет писать программы на популярной мобильной операционной системе Android $[10,11]$ и использовать в обучающем процессе $[10,12]$. Асtion Script был выбран прежде всего из-за возможности отображения большого количества объектов на экране [13] и учета взаимодействий между объектами [14].

\section{Программная реализация классов частиц}

В программе в качестве движущихся и меняющих свои свойства объектов выступают частицы. Частицы, как было отмечено выше, взаимодействуют с газовым потоком, соударяются или слипаются между собой, добавляются и удаляются из колонного реактора.

Программная реализация класса частиц угольного топлива включает три функции: инициализация, нормализация, выгорание летучих. Наиболее интересной функцией является выгорание летучих веществ. Оно происходит непрерывно, при этом углерод и газовые составляющие из частицы угольного топлива выгорают с заданной постоянной скоростью, а шлаковые составляющие остаются (поэтому 
масса шлаковых составляющих в частице будет увеличиваться по мере выгорания летучих и углерода).

Функция расчета масс выгорания летучих имеет четыре входных параметра:

function lety2 (o, pro_cMe, _0, maso2), где о - объект-частица (содержит информацию о радиусе плотности, массовых долях исходной частицы); pro_cMe - доля углерода, которая перейдет в частицу металла; _ - логическая переменная для определения начального состояния; maso2 - массовая доля кислорода в слое.

В функции выгорания рассчитывается масса целой частицы по радиусу, плотности и коэффициенту объема: $m=\frac{4}{3} \pi r^{3} \rho k_{v}$, где $m-$ масса частицы, кг; $r$ - радиус частицы, м; $\rho-$

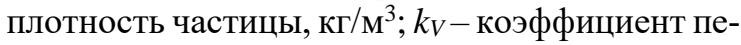
ресчета объема из программного в реальный.

Масса углерода находится по формуле $m_{\mathrm{C}}=m \chi_{\mathrm{C}}\left(1-\chi_{\mathrm{C}-\mathrm{Fe}}\right)$, где $m_{\mathrm{C}}-$ масса углерода в частице, кг; $\chi_{\mathrm{C}}$ - массовая доля углерода; $\chi_{\mathrm{C}-\mathrm{Fe}}$ - массовая доля углерода, перешедшая в частицу металла.

Затем рассчитанная масса углерода догорает до оксида углерода: $m_{\text {со_c }}=m_{\mathrm{C}} \frac{28}{12}$.

Массовые доли газовых составляющих рассчитываются по формуле $m_{\Gamma_{i}}=m \chi_{\Gamma_{i}}$, где $m_{\Gamma i}-$ масса газовой составляющей, кг; $\chi$ гі - доля газовой составляющей.

На этом этапе определяются массы углерода и летучих веществ в частице угольного топлива. Процесс выгорания летучих лимитируется содержанием кислорода, поэтому были рассмотрены три варианта, при которых выгорают только летучие компоненты топлива, летучие и углерод топлива (до СО) при недостатке кислорода, летучие и углерод до диоксида углерода.

В результате выполнения функции возвращается масса кислорода, необходимая для химических реакций, а также определяются масса и состав газов, которые будут выделены из частицы в газовую среду.

Для всех частиц угольного топлива сначала определяется газовый слой, в котором они будут выгорать, а затем в зависимости от количества кислорода в слое происходит восстановление по трем вариантам, представленным выше.

\section{Реализация класса газа}

Рассмотрим реализацию класса газа в колонном реакторе. Пространство колонного ре- актора делится на 30 слоев. Это связано с тем, что скорость газа в колонном реакторе составляет $4 \mathrm{M} / \mathrm{c}$. На следующем кадре или итерации выполнения программы слои передвигаются на один слой выше. Самый верхний слой и его состав газа удаляются. Самый нижний слой заполняется составом газа из реактора-осциллятора (рис. 1).

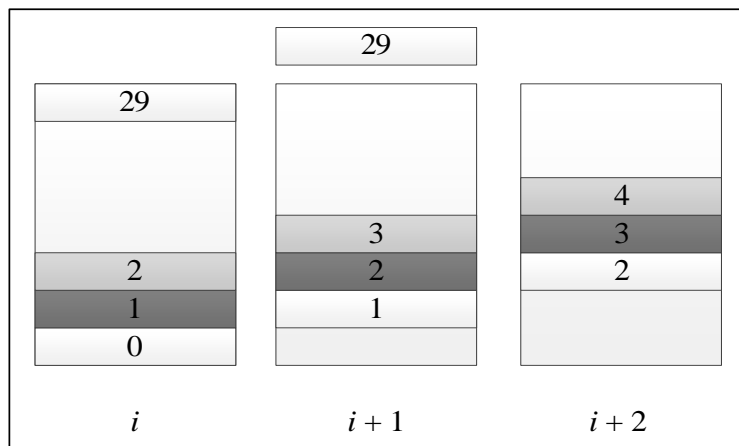

Рис. 1. Изменение газовых слоев по высоте колонного реактора

Fig. 1. Change of gas layers along the column reactor height

На рисунке показано, что с каждой итерацией происходит подъем газового слоя и удаляется 29-й слой. Состав газового слоя меняется в зависимости от того, на какой высоте выгорают частицы угольного топлива и сколько на данный момент в нем кислорода.

У класса газа по высоте есть такие свойства, как идентификатор, массовые доли газов $\mathrm{O}_{2}$, $\mathrm{CO}, \mathrm{CO}_{2}, \mathrm{CH}_{4}, \mathrm{~N}_{2}, \mathrm{H}_{2} \mathrm{O}$ и масса слоя. В соответствующем классе реализованы функции инициализации, нормализации, подъема газового слоя и определения нового состава газа.

Функция подъема газа меняет идентификаторы слоев от 0 до 29, как показано на рисунке 1. Функция определения нового состава нужна для добавления газовых составляющих из углеродного топлива и уменьшения кислорода в этом слое.

После изменений в газовом слое изменяется радиус частицы углеродного топлива, то есть происходит его уменьшение.

Расчет изменения радиуса реализован в классе частиц в виде функции

function changeR(_id, onlylety).

Входными параметрами функции являются идентификатор частицы, а также логический параметр, который при значении «Истина» реализует 1-й вариант (выгорают только летучие), при значении «Ложь» - 2-й вариант (выгорают летучие и углерод). 
Расчетные формулы для частицы углерода, у которой выгорают только летучие:

$$
m_{\text {lety }, i}=m_{\text {lety, } i-1}-\Delta m_{\text {lety, }, i}
$$

где $m_{\text {lety }}$ - общая масса летучих в частице углерода, кг; $\Delta m_{\text {lety }}$ - масса выгоревших летучих, кг.

При этом со временем уменьшается общая масса летучих.

Стоит отметить, что в частице углеродного топлива выделены три составляющие: углерод, шлаковые составляющие и летучие. Все они имеют массу и плотность.

Если будут выгорать только летучие в частице углеродного топлива, плотность частицы определится по формуле

$$
\rho=\frac{m_{\text {slak }} \cdot \rho_{\text {slak }}+m_{\mathrm{C}} \cdot \rho_{\mathrm{C}}+m_{\text {lety }} \cdot \rho_{\text {lety }}}{m_{\text {slak }}+m_{\mathrm{C}}+m_{\text {lety }}},
$$

где $m_{\text {slak }}, m_{\mathrm{C}}, m_{\text {lety }}$ - соответственно массы шлаковой составляющей, углерода и летучих, кг; $\rho_{s l a k}, \rho_{\mathrm{C}}, \rho_{\text {lety }}$ - плотности шлаковой составляющей, углерода и летучих, кг $/ \mathrm{M}^{3}$.

Радиус получившейся частицы определяется по формуле

$$
r=\sqrt[3]{m / \rho /\left(\frac{4}{3} \pi\right)}
$$

где $m=m_{\text {slac }}+m_{\mathrm{C}}+m_{\text {lety }}-$ новая масса углеродного топлива с учетом выгорания летучих, кг.

Если будет выгорать и углерод, то необходимо учесть изменение массы углерода в углеродном топливе:

$$
m_{\mathrm{C}, i}=m_{\mathrm{C}, i-1}-\Delta m_{\mathrm{C}, i}
$$

а также использовать формулы (1)-(3) для расчетов.
Если рассматривать объединенную частицу железной руды и углеродного топлива, то в ней по сравнению с формулой (2) также учитываются масса и плотность железной руды:

$$
\rho=\frac{m_{\text {ruda }} \cdot \rho_{\text {ruda }}+m_{\text {slak }} \cdot \rho_{\text {slak }}+m_{\mathrm{C}} \cdot \rho_{\mathrm{C}}+m_{\text {lety }} \cdot \rho_{\text {lety }}}{m_{\text {ruda }}+m_{\text {slak }}+m_{\mathrm{C}}+m_{\text {lety }}},
$$

где $m_{\text {ruda }}$ - масса руды, кг; $\rho_{\text {ruda }}-$ плотность руды, кг/м².

Если будут выгорать только летучие, то для расчетов в объединенной частице руды и углеродного топлива последовательно применяются формулы (1), (5), (3).

Если будут выгорать летучие и углерод, то используются формулы (1), (4), (5), (3).

\section{Результаты моделирования}

Результаты моделирования с использованием имитационной модели отражены на рисунке 2. Частицы угольного топлива сначала располагаются внизу колонного реактора, постепенно из них выгорают летучие и углерод, затем частицы углеродного топлива уменьшаются и под действием подъемной силы перемещаются вверх.

Как показали результаты моделирования, только частицам, располагающимся до 1 м по высоте колонного реактора, хватает кислорода, следовательно, углерод в частице углеродного топлива на этой высоте выгорает.

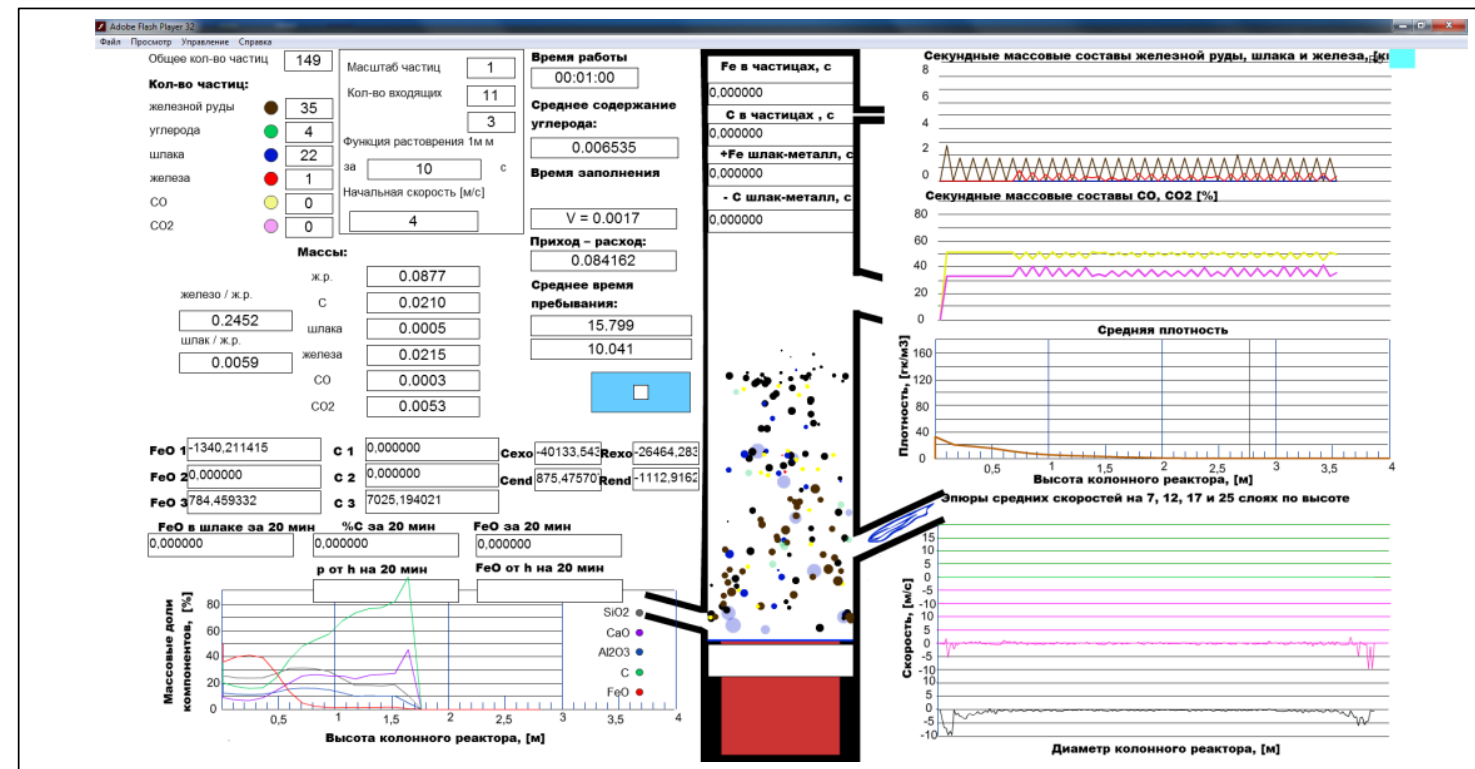

Рис. 2. Интерфейс программы многофазных многокомпонентных систем в потоке газа

Fig. 2. Program interface of multiphase multicomponent systems in gas flow 
Интерфейс программы, представленный на рисунке 2, позволяет

- задавать входные данные (количество подаваемых частиц, скорость потока газа, скорость расплавления частиц);

- отображать в режиме реального времени перемещение частиц в пространстве реактора;

- оценивать и отслеживать информацию о массовых долях компонентов, секундных массовых составах (железной руды, шлака, железа, газов), плотности по высоте реактора.

На рисунке 3 показан график среднего содержания основных газовых составляющих кислорода, угарного и углекислого газов. Содержание кислорода резко падает с 35 до $3 \%$ уже на первом метре высоты. По сравнению с начальными значениями содержание $\mathrm{CO}_{2}$ повышается, а СО уменьшается.

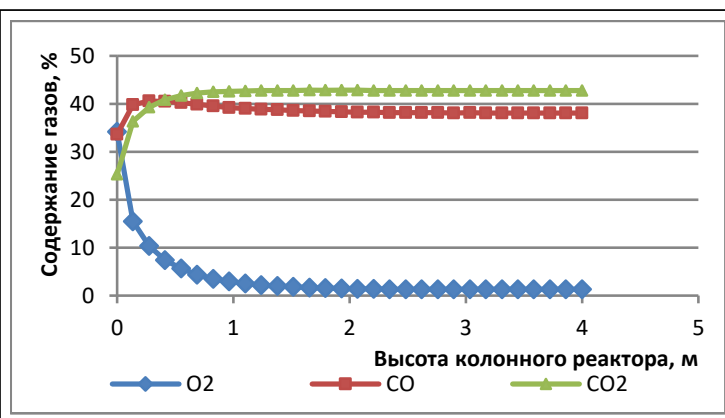

Рис. 3. Среднее содержание газов по высоте колонного реактора

Fig. 3. Average content of gases over the column reactor height

\section{Выводы}

Реализована схема горения угольного топлива, в которой на каждом шаге учитываются выделение и горение летучих и углерода при наличии кислорода в слое колонного реактора. В результате реализации такой схемы выяснилось, что не хватает кислорода для полного выгорания углерода в частицах углеродного топлива. Часть углерода вылетает вместе с частицами в шлаковое отверстие. На основе реализации схемы состава газовых слоев по высоте реактора получены данные о содержании газовых составляющих $\mathrm{O}_{2}, \mathrm{CO}$ и $\mathrm{CO}_{2}$.

Объем угольного топлива и реакции горения на его основе влияют на химические реакции и концентрацию веществ в колонном реакторе, а в итоге и на состав конечного продукта.

Разработанная модель позволит оптимально управлять сырьевыми и топливно-энергетическими ресурсами [15] в металлургическом производстве, для чего в дальнейшем потребуется учитывать процессы нагрева, плавления веществ в колонном реакторе, а также эндотермические и экзотермические реакции на уровне частиц.

Модель может найти применение в металлургии и теплоэнергетике при описании процессов в реакторах, где происходят восстановительные процессы и процессы горения топлива.

\section{Лuтература}

1. Цымбал В.П., Сеченов П.А., Рыбенко И.А. Имитационное моделирование на основе «первых принципов» и статистическая механика Гиббса // Вестн. СибГИУ. 2020. Т. 32. № 2. С. 54-67.

2. Сеченов П.А. Сравнение быстродействия алгоритмов генерации гамма-распределения для имитационной модели колонного струйно-эмульсионного реактора // Вестн. кибернетики. 2020. Т. 40. № 4. C. $42-49$.

3. Роменец В.А. Процесс Ромелт. М.: МИСиС; Руда и металлы, 2005. 400 с.

4. Черменев Е.А. Исследование процессов нагрева и плавления окатышей в ванне дуговой печи с целью повышения энергоэффективности электроплавки стали: автореф. дис. М.: НИТУ МИСиС, 2014. $28 \mathrm{c}$.

5. Ермакова Л.А., Мочалов С.П., Калашников С.Н., Пермяков А.А. Механизм горения капель суспензионного водоугольного топлива в вихревой топке автоматизированного энергогенерирующего комплекса // Вестн. КемГУ. 2012. Т. 2. № 4. С. 164-169.

6. Ермакова Л.А. Математическое моделирование процесса горения капель водоугольного топлива // Междисциплинарные исследования в области математического моделирования и информатики: матер. 5-й науч.-практич. internet-конф. 2015. С. 173-178.

7. Бесценный И.В., Щудло Т.С., Дунаевская Н.И., Топал А.И. Исследование особенностей горения смесей коксов углей различной степени метаморфизма и коксов биомассы // Теплоэнергетика. 2013. № 12. C. 4-8.

8. Пинчук В.А. Влияние характеристик водоугольного топлива на закономерности процесса его горения // Современная наука: исследования, идеи, результаты, технологии. 2014. № 2. С. 78-84. 
9. Vechi A., Brito A.F., Valentim D.B., Gozzi M.E., Sampaio A.R., Viscovin R.C. Dynamic model of the Solar System in actionscript with control ranges to astronomy and astrophysics teaching. Revista Brasileira de Ensino de Física, 2013, vol. 35, no. 2, pp. 1-25. DOI: 10.1590/S1806-11172013000200025.

10. Rozali N.F., Zaid N.M. Code puzzle: ActionScript 2.0 learning application based on problem based learning approach. Proc. 6th ICT-ISPC, 2017, pp. 1-4. DOI: 10.1109/ICT-ISPC.2017.8075329.

11. Samsudin S., Irawan M.D., Harahap A.H. Mobile app education gangguan pencernaan manusia berbasis multimedia menggunakan Adobe Animate CC. Jurnal Teknologi Informasi, 2019, vol. 3, no. 2, pp. 141-148. DOI: 10.36294/JURTI.V3I2.1009.

12. Беспалько А.А., Сочнева Н.В. Возможности программного продукта Adobe Flash CS5 для создания обучающего курса по информатике // Педагогика и современность. 2012. № 1. С. 138-141.

13. Мук К. ActionScript 3.0 для Flash; [пер. с англ.]. СПб: Питер, 2009. 992 с.

14. Сеченов П.А., Цымбал В.П., Оленников А.А. Постановка задачи исследования диффузионного перехода через границу шлак-металл в колонном реакторе и алгоритм ее решения // Программные продукты и системы. 2016. Т. 29. № 4. С. 156-164. DOI: 10.15827/0236-235X.116.156-164.

15. Спирин Н.А., Онорин О.П., Гурин И.А., Лавров В.В., Щипанов К.А. Структура системы оптимального управления сырьевыми и топливно-энергетическими ресурсами в доменном производстве // Теплотехника и информатика в образовании, науке и производстве: сб. докл. VII Всерос. науч.-практич. конф. 2018. С. 526-536.

\title{
Simulation of multiphase multicomponent systems in a gas flow
}

P.A. Sechenov ${ }^{1}$, Ph.D. (Engineering), pavesa89@mail.ru

I.A. Rybenko ${ }^{1}$, Dr.Sc. (Engineering), Associate Professor, rybenkoi@mail.ru

V.P.Tsymbal ${ }^{1}$, Dr.Sc. (Engineering), Professor, tsymbal33@mail.ru

\author{
${ }^{1}$ Siberian State Industrial University, Novokuznetsk, 654007, Russian Federation
}

Abstract. The paper considers a simulation model of physical and chemical processes in a column jetemulsion reactor. The tables show the initial compositions of the input products: iron ore, lime, coal fuel.

Computational experiment involves using a simulation model and a particle method. The author propose a technique of the interaction of particles in a column reactor, considers the mechanisms of interaction and changes in the gas composition along the reactor height in detail. The chosen programming language is ActionScript 3.0 that allows animating the movement of particles and visual studying the ongoing processes. It was compiled in the Adobe Animate programming environment. The mechanism of coal fuel combustion is implemented, and the options of volatile burnup in coal fuel are taken into account depending on the presence of oxygen in gas layer. The properties in coal fuel particles dynamically change as they burn out: the size decreases, the composition changes and the density increases. To take into account the change in the gas composition along the column reactor height, a corresponding class is implemented; and a mechanism for gas rise and gas composition change in the form of layers along the reactor height is proposed.

As a result of modeling, it was revealed that when the coal fuel burns out and the properties change, the particles begin to rise up the column reactor. The highest concentration of coal fuel fine particles is observed just below the level of the slag taphole, where particles of slag and coal fuel fly out. The modeling also showed that the oxygen concentration drops sharply already at the first meter along the reactor height, the carbon monoxide concentration slightly decreases, and the carbon dioxide concentration increases.

The developed and implemented model will allow optimal control of raw materials, fuel and energy resources in metallurgical production. In the future, it will be necessary to take into account the processes of heating, substance melting in the column reactor, as well as endothermic and exothermic reactions at the particle level.

Keywords: column reactor, thermodynamics, simulation model, particle interaction, algorithm, software implementation. 


\section{References}

1. Tsymbal V.P., Sechenov P.A., Rybenko I.A. Simulation on the basis of "first principles" and the statistical mechanics of Gibbs. Bull. of the SibSIU, 2020, vol. 32, no. 2, pp. 54-67 (in Russ.).

2. Sechenov P.A. Speed comparison of gamma distribution generation algorithms for simulation model of column jet-emulsion reactor. Proceedings in Cybernetics, 2020, vol. 40, no. 4, pp. $42-49$ (in Russ.).

3. Romenets V.A. Romelt Process. Moscow, 2005, 400 p. (in Russ.).

4. Chermenev E.A. Investigation of the Pellet Heating and Melting Processes in the Arc Furnace Bath an in Order to Increase the Energy Efficiency of Electric Steel Melting. Ph. D. Thesis, Moscow, National University of Sciense and Technology MISIS, 2014, 28 p. (in Russ.).

5. Ermakova L.A., Mochalov S.P., Kalashnikov S.N., Permyakov A.A. Mechanism of coal-water fuel drops combustion in the swirl burner of an automated energy generating complex. Bull. of Kemerovo State University, 2012, vol. 2, no. 4, pp. 164-169 (in Russ.).

6. Ermakova L.A. Mathematical modeling of combustion processes drops coal-water slurry. Proc. 5th Internet Conf. Interdisciplinary Research in Mathematical Modeling and Computer Science, 2015, pp. 173-178 (in Russ.).

7. Bestsennyi I.V., Shchudlo T.S., Dunaevskaya N.I., Topal A.I. Studying the specific features pertinent to combustion of chars obtained from coals having different degrees of metamorphism and biomass chars. Thermal Engineering, 2013, vol. 60, no. 12, pp. 853-858. DOI: 10.1134/s0040601513120033.

8. Pinchuk V.A. The influence of various physical and chemical parameters of coal-water fuel on the characteristics of its combustion process. MODERN SCIENCE: Researches, Ideas, Results, Technologies, 2014, vol. 15, no. 2, pp. 78-84 (in Russ.).

9. Vechi A., Brito A.F., Valentim D.B., Gozzi M.E., Sampaio A.R., Viscovin R.C. Dynamic model of the Solar System in actionscript with control ranges to astronomy and astrophysics teaching. Revista Brasileira de Ensino de Física, 2013, vol. 35, no. 2, pp. 1-25. DOI: 10.1590/S1806-11172013000200025.

10. Rozali N.F., Zaid N.M. Code puzzle: ActionScript 2.0 learning application based on problem based learning approach. Proc. 6th ICT-ISPC, 2017, pp. 1-4. DOI: 10.1109/ICT-ISPC.2017.8075329.

11. Samsudin S., Irawan M.D., Harahap A.H. Mobile app education gangguan pencernaan manusia berbasis multimedia menggunakan Adobe Animate CC. Jurnal Teknologi Informasi, 2019, vol. 3, no. 2, pp. 141-148. DOI: $10.36294 / J U R T I . V 3 I 2.1009$

12. Bespalko A.A., Sochneva N.V. Features of Adobe Flash CS5 software for creating a computer science training course. Pedagogy and Modernity, 2012, no. 1, pp. 138-141 (in Russ.).

13. Moock C. Essential ActionScript 3.0. O’Rielly Media Publ., 2007, 911 p. (Russ. ed.: St. Petersburg, 2009, 992 p.).

14. Sechenov P.A., Tsymbal V.P., Olennikov A.A. Statement of the research problem of the diffusion transition across the slag-metal border in a column reactor and its solution algorithm. Software and Systems, 2016, vol. 29, no. 4, pp. 156-164. DOI: 10.15827/0236-235X.116.156-164 (in Russ.).

15. Spirin N.A., Onorin O.P., Gurin I.A., Lavrov V.V., Shchipanov K.A. The structure of the system for optimal management of raw materials and fuel and energy resources in blast-furnace production. Proc. 7th AllRus. Sci.-Pract. Conf. Heat Engineering and Informatics in Education, Science and Production, 2018, pp. 526-536 (in Russ.).

\section{Для цитирования}

Сеченов П.А., Рыбенко И.А., Цымбал В.П. Имитационное моделирование многофазных многокомпонентных систем в потоке газа // Программные продукты и системы. 2021. Т. 34 . № 3. C. 477-483. DOI: $10.15827 / 0236-235 X .135 .477-483$.

\section{For citation}

Sechenov P.A., Rybenko I.A., Tsymbal V.P. Simulation of multiphase multicomponent systems in a gas flow. Software \& Systems, 2021, vol. 34, no. 3, pp. 477-483 (in Russ.). DOI: 10.15827/0236235X.135.477-483. 